
\title{
25 Research Soure \\ Benefits of a standardized protocol for axillary management after neoadjuvant chemotherapy in a single center.
}

Marina de Paula Canal ( $\triangle$ mpcana738@gmail.com )

ACCamargo Cancer Center https://orcid.org/0000-0002-6052-6245

Caroline gomes de almeida Rocha

ACCamargo Cancer Center

Cynthia Ap. Bueno de Toledo Osório

ACCamargo Cancer Center

Almir Bitencourt

ACCamargo Cancer Center

Marina Sonagli

ACCamargo Cancer Center

Monique Celeste Tavares

ACCamargo Cancer Center

Solange Moraes Sanches

ACCamargo Cancer Center

Fabiana Baroni Alves Makdissi

ACCamargo Cancer Center

\section{Research Article}

Keywords: Axillary lymph node, neoadjuvant chemotherapy, downstaging, standardized protocol, clip marker

Posted Date: April 28th, 2021

DOl: https://doi.org/10.21203/rs.3.rs-395622/v1

License: (c) (i) This work is licensed under a Creative Commons Attribution 4.0 International License. Read Full License 


\section{Abstract}

Purpose: Axillary lymph node status is one of the most important prognostic factors in breast cancer. Neoadjuvant chemotherapy (NACT) has been indicated for locally advanced tumors, and for HER2positive and triple negative tumors larger than 2.0 and $1.0 \mathrm{~cm}$, respectively, regardless of axillary status. NACT allows clinicians to assess the biological responses of the tumor, predicting prognosis and permitting more conservative surgeries in breast and axilla.

Objective: evaluate the use of a standardized protocol after NACT to enable, sentinel node dissection (SLND) in patients with axillary downstaging, avoiding axillary dissection (ALND).

Methods: retrospective cohort study of data collected from medical records of patients who underwent NACT in a single center, from January 2014 to December 2018. 471 patients were included in this study: 303 patients before and 165 patients after the implementation of this protocol. This protocol comprises a placement of a metal clip in biopsied lymph node, in patients with up to 2 clinically lymph nodes. After NACT, if a radiologic complete response were achieved, SLND was performed using blue die and tecnecion99. ALDN were avoided in patients whose clipped sentinel node were negative for metastasis. Trial registration number $2786 / 19$, october, 19,2019 , retrospectively registered.

Results: Protocol avoided ALDN in $18 \%$ of selected patients $(p<0.05)$. Also, patients with triple negative and HER2 positive tumors underwent SLND most frequently, when compared to Luminal tumors ( $p$ 0.03). This results showed that the proposed protocol can improve SLND in selected patients when axillary targeted dissection cannot be performed.

\section{Highlights}

- Axillary lymph node status is one of the most important prognostic factors in breast cancer.

- Neoadjuvant chemotherapy has been indicated for locally advanced tumors, and for HER2-positive and triple negative tumors larger than 2.0 and $1.0 \mathrm{~cm}$, respectively, regardless of axillary status.

- A standardized protocol was created with the objective of reducing the number of false-negative results of sentinel lymph node dissection and minimizing technical limitations of target axillary dissection.

\section{Introduction}

Axillary lymph node status is one of the most important prognostic factors in breast cancer. For locally advanced tumors, neoadjuvant chemotherapy (NACT)[1] shows no difference in overall survival (OS)and specific-cancer survival(SCS) when compared to adjuvant chemotherapy [2], but it favors higher rates of lumpectomy of the breast and axilla [3]. In addition, the current indication for NACT in breast cancer 
allows for in vivo evaluation of the tumor for systemic treatment, which has an important prognostic value for certain subtypes, such as triple negative and HER2-positive.

Axillary lymph node dissection (ALND) is related to an increased risk of adverse events, such as lymphedema (14\%), limitation of upper limb mobility (28\%), and neuropathic pain (31\%)[4]. Nevertheless, it remains the gold standard for treatment of axillary lymph node tumors, even after complete clinical / radiological response to NACT[1].

The three main clinical, prospective, randomized studies that assessed axillary management after NACT(ACOSOG Z1071, SENTINA arm C, and SN FNAC)[5-7] mainly included CT1, cT2, and cN1 patients who underwent sentinel lymph node dissection (SLND), followed by ALND, after NACT. The detection of sentinel lymph nodes (SLNs) was possible in $80-92.7 \%$ ofcN+ patients who had a clinical response. The false negative rate (FNR) ranged from $12.3 \%$ to $14.2 \%$. However, when 3 more SLNs were removed the FNR dropped to $4.9-9.1 \%$ (using technetium 99 detection method) and $8.6-10.8 \%$ (using blue dye).

The strategy of target axillary dissection has been adopted as a strategy to reduce the FNR in $\mathrm{cN}+$ patients. In this technique, the target lymph node is marked with a metal clip at the time of biopsy, before NACT and up to five days before surgery. An additional ultrasound (US) is then performed, during which the clipped lymph node receives radioactive 'seeds' [8] and technetium 99 (Tc99) and blue dye, ensuring accurate SLND. With this technique, FNRs as low as $2 \%$ have been observed, and in $77 \%$ of cases the marked lymph node corresponded to the SLN [9]. However, target axillary dissection has not been universally adopted because of difficulties related to pre- and intra- operative localization of previously marked lymph nodes which had shown complete response to NACT[1]. In view of this, to minimize the FNR in relation to axillary dissection, we have developed a standardized protocol at our institution for clipping positive lymph nodes prior to NACT combined with post-NACT axillary management. The objective of this study is to show that it is possible to prevent ALND in clinically negative patients after NACT, using a protocol that is easily accessible to doctors and patients from other centers.

\section{Materials And Methods}

This was a retrospective cohort study of data collected from medical records of patients who underwent NACT in a single center, from January 2014 to December 2018. This study was approved by institutional ethics review board. Data collected included molecular subtype of the tumor, clinical stage ( $T$ or $N)$ prior to NACT, type of surgery (mastectomy or lumpectomy), and type of axillary dissection (ALND, SLND, or SLND followed by ALND). Patients were included if they had 1) been diagnosed with invasive breast carcinoma and were undergoing NACT, 2) undergone biopsy or slide review at the institution, and 3) received all treatment (chemotherapy and surgery) at the institution. Patients were excluded if they 1 ) were male, or 2) had inflammatory, metastatic, recurrent, or bilateral tumors.

Patients were examined before and after the institution of a standardized protocol for axillary treatment after NACT. There were three possible protocols, depending on the lymph node status of each patient: axilla clinically negative (Fig 1 a); up to two clinically positive lymph nodes prior to NACT, which were clipped (Fig 1 b); up 
to two clinically positive lymph nodes prior to NACT, which were not clipped (Fig $1 \mathrm{c}$ ). In short, ALND was performed in patients with two or more clinically positive lymph nodes prior to NACT, patients with persistent disease after NACT (clinical or radiological), and patients whose initial stage was T4 or inflammatory and had no SLN migration. This was followed by SLND in patients with up to two clinically positive lymph nodes prior to NACT, and patients who had had a complete clinical and radiological response after NACT and SLN migration.

SLN marking was performed on the eve of surgery through injection of Tc99 into the breast, close to the tumor area, with lymphoscintigraphy to evaluate migration. Marking with blue dye was performed during surgery through a subdermal injection into the ipslateral breast in the periareolar or superolateral quadrant, depending on the surgeon's preference. When removing a lymph node marked by Tc99 or blue dye (Fig 2), a portable X-ray (Faxitron ${ }^{\circledR}$ ) confirmed the presence of the clip for protocol validation (Fig 3).

For statistical analysis, descriptive statistical methods were used and the results of categorical variables were expressed as frequencies and percentages. Correlation analysis was performed using Pearson's chisquare test with Yates correction or Fisher's exact test, when indicated. The level of significance adopted was $5 \%(\mathrm{p} \leq 0.05)$.3. Results A total of 471 female patients were analyzed, 303 patients before and 165 patients after the implementation of this protocol, 03 patients were excluded due to missing data on medical record, aged 24-87 years, divided according to TNM staging (cT1-cT4, cN0,cN+) and molecular subtype (Luminal A and B HER2negative, Luminal HER2positive and HER2hyperexpressed, and triple negative)(Table 1).

Table 1: Characteristics of patients according to TNM staging and molecular subtype.

\begin{tabular}{|c|c|c|c|}
\hline \multicolumn{2}{|c|}{$\mathrm{T}$ and $\mathrm{N}$ and molecular subtype } & $\mathbf{N}$ & $\%$ \\
\hline \multirow[t]{5}{*}{$\mathrm{T}$} & cT1 & 36 & 7,7 \\
\hline & cT2 & 173 & 37,0 \\
\hline & сT3 & 141 & 30,2 \\
\hline & cT4 & 117 & 25,1 \\
\hline & Total & 467 & 100,0 \\
\hline \multicolumn{2}{|l|}{ No data } & 4 & \\
\hline \multirow[t]{3}{*}{$\mathbf{N}$} & No & 76 & 16,5 \\
\hline & $\mathrm{N}+$ & 385 & 83,5 \\
\hline & Total & 461 & 100,0 \\
\hline No data & & 10 & \\
\hline \multirow[t]{4}{*}{$\begin{array}{l}\text { Molecular } \\
\text { subtype }\end{array}$} & $\begin{array}{l}\text { Luminal A and Luminal B HER2 negative and Luminal (Ki67 14-20\%, RP > 20\%) } \\
\text { HER2 negative }\end{array}$ & 210 & 44,9 \\
\hline & Luminal B HER2 positive plus hyperexpress HER2 & 122 & 26,1 \\
\hline & Triple negative & 136 & 29,1 \\
\hline & Total & 468 & 100,0 \\
\hline No data & & 3 & \\
\hline Total & & 471 & $100 \%$ \\
\hline
\end{tabular}


Of these patients, 295 underwent mastectomy (simple, modified radical, or skin-sparing) and 176 underwent breast-conserving surgeries (quadrantectomy or lumpectomy). ALND was performed in 303 patients, SLND in 156, and SLND followed by ALND in nine.

In the period from 2014 to 2017, prior to the implementation of a standardized protocol for preNACT axillary management, 303 patients were included (Fig 4).

Beginning in 2017, a standardized protocol for pre-NACT axillary management was instituted, from which time 165 patients were included (Fig 5).

Sixty-seven of these patients were axillary clinically negative, 49 of whom underwent SLND or SLND followed by ALND, and 18 of whom underwent ALND. The remaining 98 patients, who were axillary clinically positive, were submitted to fine needle aspiration biopsy to test for malignancy. Of the 24 patients who tested negative for malignancy, sixteen underwent SLND and eight underwent ALND. Of the 74 who tested positive for malignancy, 43 underwent ALND, 24 underwent SLND, and 7 underwent SLND followed by ALND Prior to implementation of the standard protocol, $77.2 \%$ of patients underwent ALND and 22.8\% underwent SLND; after implementation, 59.2\% underwent ALND and $40.8 \%$ SLND. The increase in SLND after protocol implementation was statistically significant $(p<0.05)$.

Rates of SLND differed across molecular subtypes. Patients with tumors that were triple negative underwent SLND most frequently (44\%), followed by Luminal B HER2 positive with HER2 hyperexpression (32\%), and Luminal HER2 negative (26\%). The difference between triple negative and Luminal patients was significant $(p=0.03) \cdot 4$. Discussion In the present study we describe a standardized protocol for post-NACT axillary management which increases the efficacy of target axillary dissection and reduces the false negative rate of SLND. $\quad$ NACT is an important tool in the treatment of certain breast cancers because it not only reduces tumor burden by initially treating the systemic micrometastatic disease, but has also been shown to increase the rate of conservative surgeries in patients who would not otherwise be candidates [12-14] NACT also plays a role in axillary downstaging, improving outcomes of clinically positive patients who underwent chemotherapy prior to SLND. The present study similarly found that, in the ninety-eight patients who presented clinically positive axilla, NACT prevented lymphadenectomy in $46 \%$ of cases. This result is nearly identical to that of Mamtani et al., whoreported in a prospective study that $70 \%$ of clinically positive patients were eligible for SLND after NACT and $48 \%$ were able to avoid ALND [15]. Together these studies demonstrate the role of NACT in reducing the need for ALND among patients with lymph node metastases.

Considering the performance of lumpectomy after NACT, Bonadonna and Veronesi reported that NACT reduced large tumors to less than $3 \mathrm{~cm}$ in $81 \%$ of patients, allowing lumpectomy instead of radical mastectomy in $50-75 \%$ of patients for whom mastectomy was initially indicated [13-14]. The results of our study were in agreement, allowing lumpectomy in $52.6 \%$ of patients with T2 staging. Regarding molecular subtypes, we observed complete radiological response in $56 \%$ of triple negative tumors, and $54 \%$ of HER2 positive. A similar study also observed complete radiological response in $59 \%$ of cases [16]. Moreover, patients who were triple negative underwent significantly more SLND than those in the Luminal (HER2 negative) subgroup. This study has limitations inherent to its retrospective design. First, the medical records were not standardized, 
especially regarding axillary status prior to the implementation of institutional protocol in 2017. In addition, the relatively small number of patients in the sample may have limited statistical analyses. Despite this, the present study demonstrates effectiveness in implementing a standardized protocol for axillary management before and after NACT. The institutional protocol used was created with the objective of reducing the number of false-negative results of SLND and minimizing technical limitations of the target axillary dissection that make its routine implementation unfeasible, such as difficulty in locating the clipped lymph node, especially when it has a complete response to treatment [17].

In conclusion, the results of this study showed an increase in the frequency of SLND after implementation of a standardized protocol for management of axilla after NACT in breast cancer patients. Prospective studies, with a larger sample and in other centers, are necessary to validate the proposed technique.

\section{Declarations}

Funding - Not applicable

Conflicts of interest/Competing interests - None

Availability of data and material - The datasets generated during and/or analysed during the current study are not publicly available due to restrictions by our ethics committee but are available from the corresponding author on reasonable request.

Code availability - Not applicable

\section{Authors' contributions (all of them):}

- Substantial contributions to the conception or design of the work; or the acquisition, analysis, or interpretation of data for the work; AND

- Drafting the work or revising it critically for important intellectual content; AND

- Final approval of the version to be published; AND

- Agreement to be accountable for all aspects of the work in ensuring that questions related to the accuracy or integrity of any part of the work are appropriately investigated and resolved.

Ethics approval :Trial registration number 2786/19, approved in October, 19, 2019.

\section{References}

1. Nguyen TT, Hoskin TL, Day CN, Degnim AC, Jakub JW, Hieken TJ, Boughey JC, et al. Decreasing Use of Axillary Dissection in Node-Positive Breast Cancer Patients Treated with Neoadjuvant Chemotherapy. Ann SurgOncol. 2018 Sep;25(9):2596-2602 
2. Rubovszky G, Horváth Z, et al. Recent Advances in the Neoadjuvant Treatment of Breast Cancer. J BreastCancer. 2017;20(2):119-31

3. Haffty BG, McCall LM, BallmanKV, McLaughlin S, Jagsi R, Ollila DW, Hunt KK, Buchholz TA, Boughey JC, et al.Patterns of local-regional management following neoadjuvant chemotherapy in breast cancer: Results from ACOSOG Z1071 (Alliance). Int J RadiatOncolBiolPhys. 2016;1;94(3):493502.

4. Fleissig A, Fallowfield LJ, Langridge $\mathrm{Cl}$, et. al. Post-operative arm morbidity and quality of life: results of the ALMANAC randomized trial comparing sentinel node biopsy with standard axillary treatment in the management of patients with early breast cancer. Breast Cancer ResTreat. 2006;95(3):279-93

5. Boughey JC, Suman VJ, Mittendorf EA, et. al. Sentinel lymph node surgery after neoadjuvant chemotherapy in patients with node-positive breast cancer: the ACOSOG Z1071 (Alliance) clinical trial. JAMA. 2013;310(14):1455-61

6. Kuehn T, Bauerfeind I, Fehm T, et. al. Sentinel-lymph-node biopsy in patients with breast cancer before and after neoadjuvant chemotherapy (SENTINA): a prospective, multicentre cohort study. Lancet Oncol. 2013;14(7):609-18

7. Boileau JF, Poirier B, Basik M, et. al. Sentinel node biopsy after neoadjuvant chemotherapy in biopsyproven node positive breast cancer: the SN FNAC Study. J Clin Oncol. 2015:33(3):258-64

8. Pilewskie M, Morrow M, et al. Axillary Nodal Management Following Neoadjuvant Chemotherapy: A Review. JAMA Oncol. 2017;1;3(4):549-555

9. Caudle AS, Yang WT, Krishnamurthy S, et. al. Improved Axillary Evaluation Following Neoadjuvant Chemotherapy for Patients With Node-Positive Breast Cancer Using Selective Evaluation of Clipped Nodes: Implementation of Target Axillary Dissection. J Clin Oncol. 2016;34:1072-8

10. Symmans WF, Peintinger F, Hatzis C, Rajan R, Kuerer H, Valero V, Assad L, et at. Measurement of residual breast cancer burden to predict survival after neoadjuvant chemotherapy. J Clin Oncol. 2007 Oct 1;25(28):4414-22.

11. Osório C T; Soares F A, et al. Assessment of pathological response in breast cancer after neoadjuvant chemotherapy: standardization of adapted protocol. Bras. Patol. Med. Lab. vol.48 no.6 Rio de Janeiro Dec. 2012

12. Aghian A, El-Ghamry MN, Merajver SD, et al. Clinical features and management of locally advanced and inflammatory breast cancer. UpToDate Online 17.3 2009 Sep-Dec [last updated May 12].

13. Mauriac L, Durand M, Avril A, Dilhuydy JM, et al. Effects of primary chemotherapy in conservative treatment of breast cancer patients with operable tumors larger than $3 \mathrm{~cm}$. Results of a randomized trial in a single centre. Ann Oncol. 1991;2(5):347-54.

14. Bonadonna G, Veronesi U, Brambilla C, Ferrari L, Luini A, Greco M, et al.Primary chemotherapy to avoid mastectomy in tumors with diameters of three centimeters or more. J Nat Cancer Inst. 1990;82(19):1539-45. 
15. Mantani A, Barrio A, King T, Van Zee K J, Pilewskie M et al. How Often Does Neoadjuvant Chemotherapy Avoid Axillary Dissection in Patients With Histologically Confirmed Nodal Metastases? Resultsof a ProspectiveStudy .AnnSurgOncol 2016 Oct;23(11):3467-3474.

16. Batt, J, Chambers, A, Al-Allak, A, Vestey, S, Hunt, R, Massey, et al. Neo-Adjuvant chemotherapy and its affects to the axilla-Can we safely downgrade axillary surgery to mirror the approach in the breast. The Breast Journal 2020, Aug; 26(09): 1667-1672.

17. Ecanow J, Abe H, Newstead G, Ecanow D, Jeske J, et al. Axillary staging of breast cancer: what the radiologist should know. Radiographics. 2013;33(6):1589-1612.

\section{Figures}



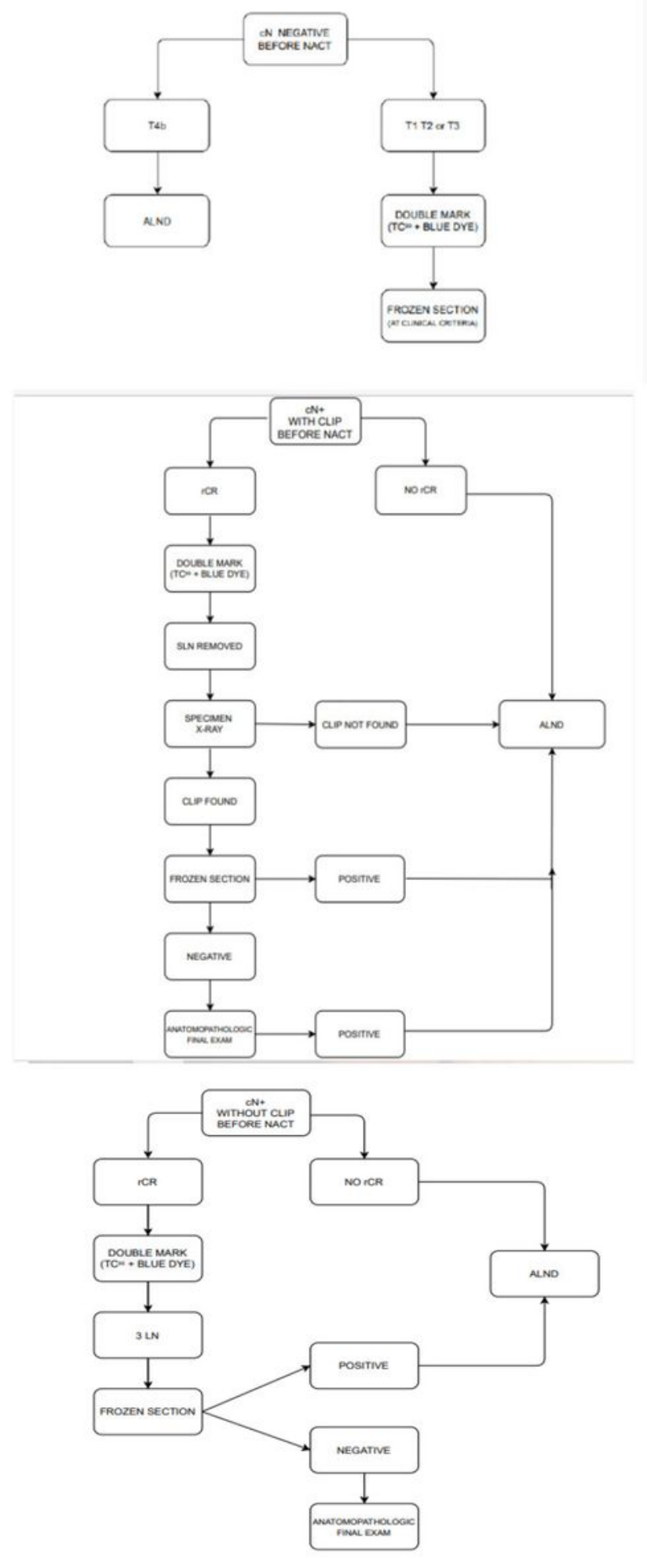

\section{Figure 1}

Top panel: A: Protocol for patients with axilla clinically negative prior to NACT. Middle panel: 1B: Protocol for patients with up to two clinically positive lymph nodes prior to NACT, which were clipped. Bottom panel: Protocol for patients with up to two clinically positive lymph nodes prior to NACT, which were not clipped. 


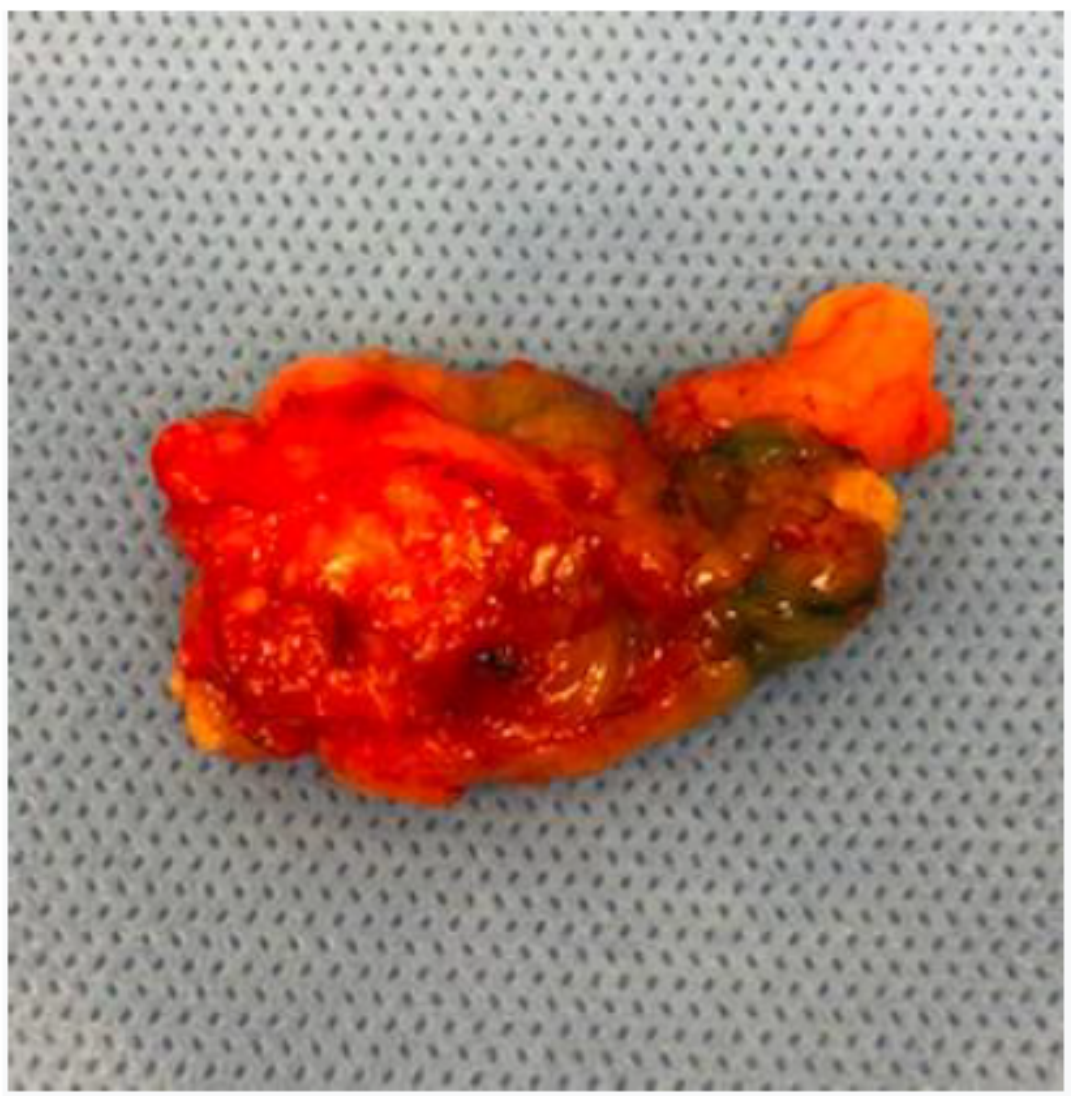

\section{Figure 2}

Photo of SLN stained with blue dye.

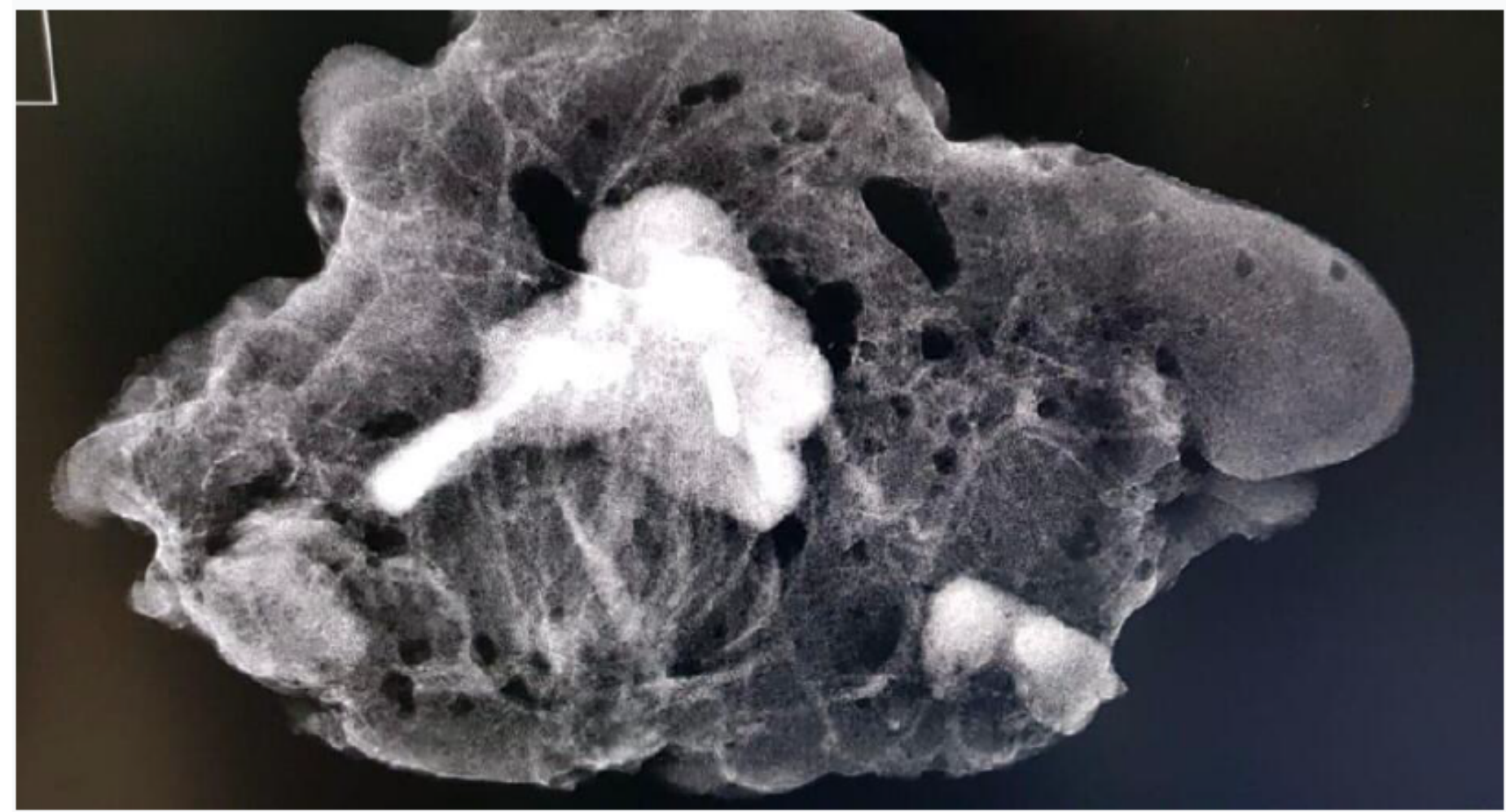




\section{Figure 3}

X-ray photo of clipped lymph node. Pathological analysis (frozen section) of lymph nodes after NACT [10\} was performed in three parts: 1- Macroscopic examination. In the perioperative examination, SLNs were sliced transversely to a thickness of $2 \mathrm{~mm}$ and examined by a pathologist for the presence of any whitish areas of hardened consistency, suggestive of residual lymph node metastasis. All slices of lymph node tissue were fixed in $10 \%$ buffered formalin and included in one or more paraffin blocks for the definitive histological evaluation. 2- Microscopic evaluation. In the microscopic evaluation, a pathologist measured the linear dimension of the largest metastatic focus and described the presence of a possible area of pathological response, characterized by fibrosis, hemorrhage, accumulations of macrophages and a decrease in the lymph node parenchyma. Additional sections of $4-\mu \mathrm{m}$ thickness were stained with hematoxylin-eosin and analyzed by a pathologist for the presence of isolated cells, or a group of atypical epithelial cells compatible with residual neoplasia, which would determine ALND. The metal clip area was also described, characterized by foreign body-type gigantocellular reaction and lymphocytic infiltrate around amorphous acidophilic material, compatible with the gel shell present in the clips used. 3- Tumor presence. The presence of axillary nodal tumor deposits of any size, including isolated tumor cells, excluded a complete pathological response. Finally, the number of compromised lymph nodes was counted and classified to obtain the rCR index and classification[11]. 


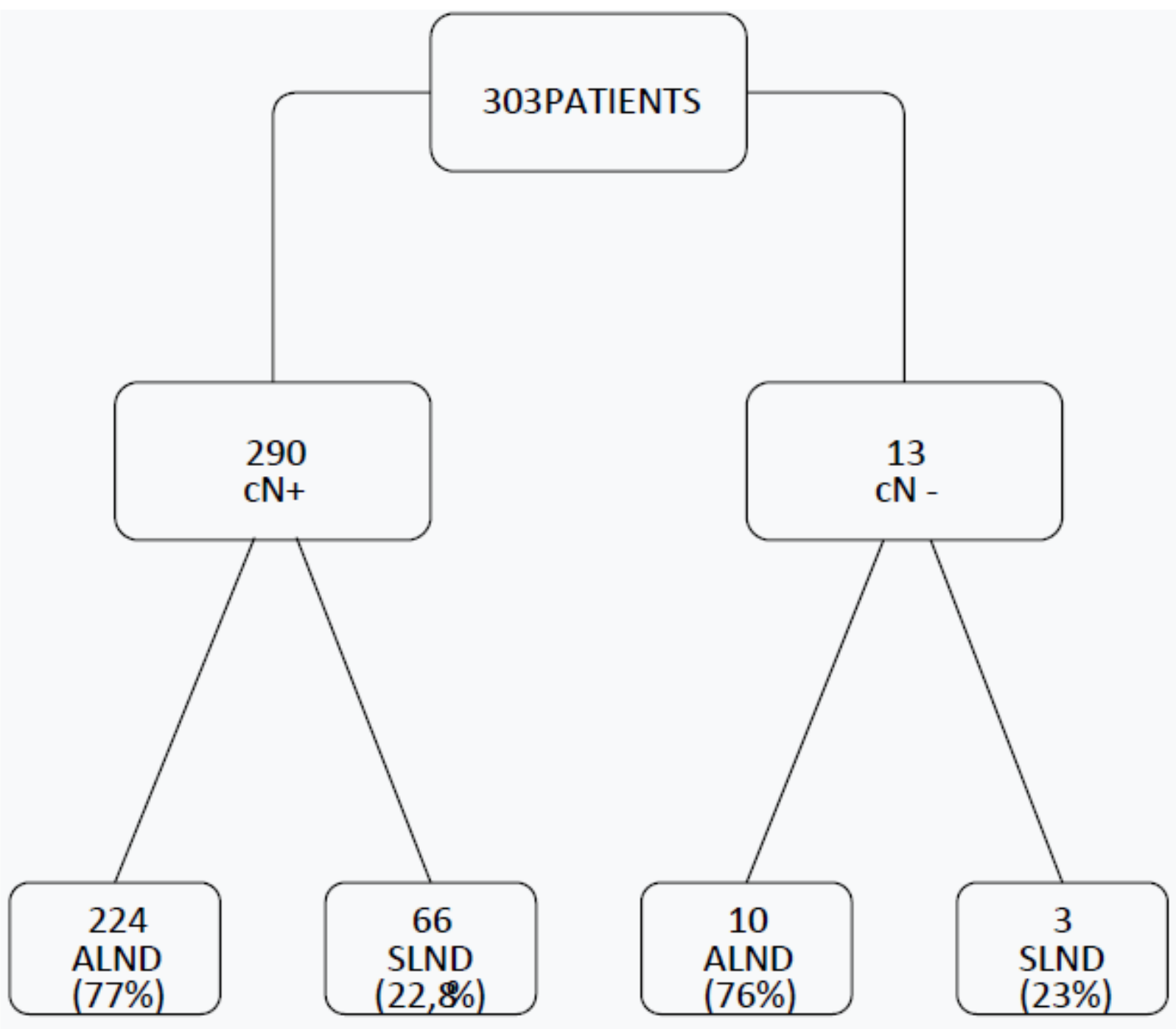

Figure 4

Division of patients who were evaluated from 2014-2017, before implementation of the standardized protocol. 


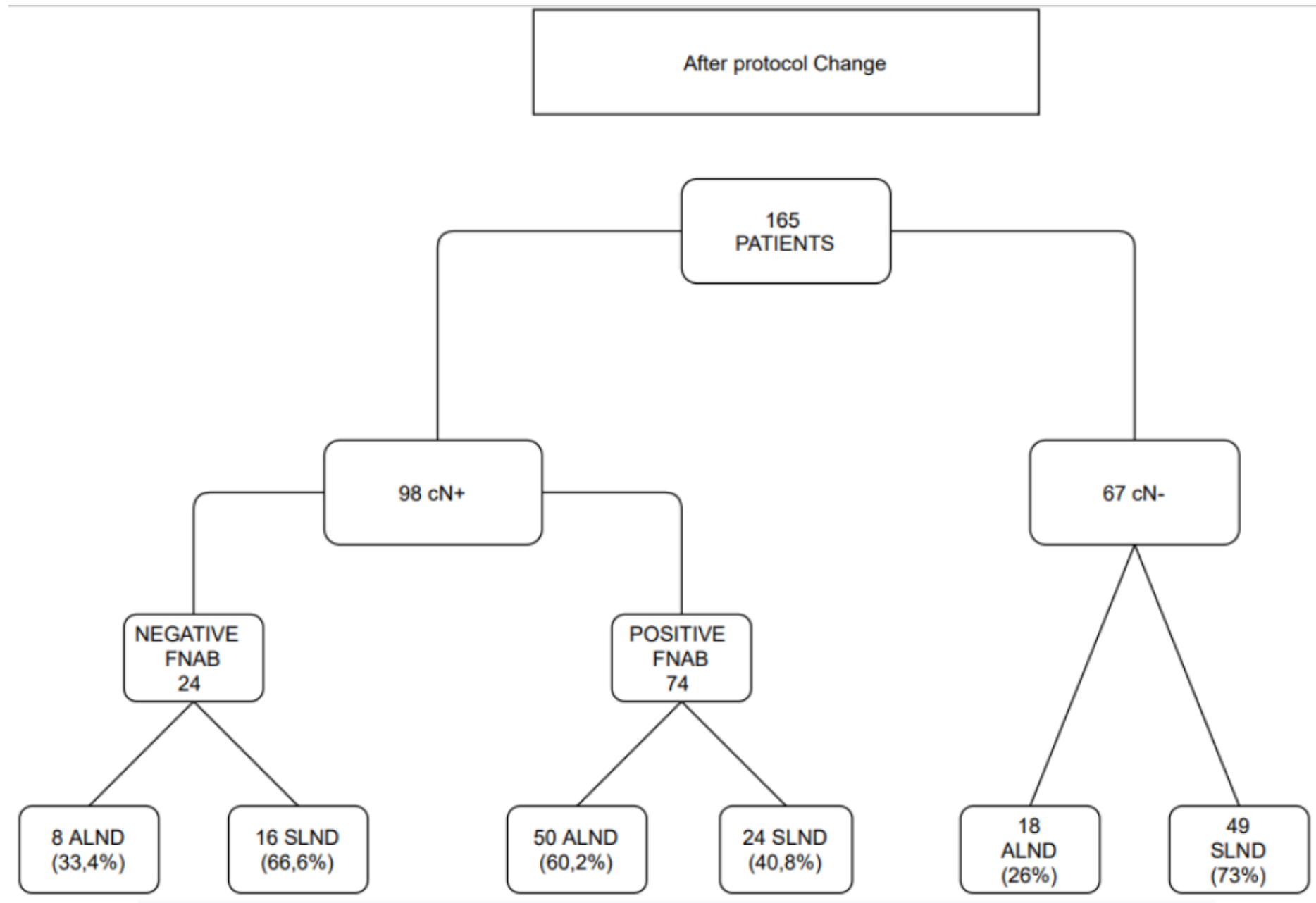

Figure 5

Description of the groups after implementation of the protocol. 